\title{
Simulation of multicorrelated random processes using the FFT algorithm
}

\author{
L. E. Wittig* and A. K. Sinha ${ }^{\dagger}$ \\ Department of Engineering Science and Mechanics, Virginia Polytechnic Institute and State University, \\ Blacksburg, Virginia 24060 \\ (Received 27 July 1973)

\begin{abstract}
A technique for the digital simulation of multicorrelated Gaussian random processes is described. This technique is based upon generating discrete frequency functions which correspond to the Fourier transform of the desired random processes, and then using the fast Fourier transform (FFT) algorithm to obtain the actual random processes. The main advantage of this method of simulation over other methods is computation time; it appears to be more than an order of magnitude faster than present methods of simulation. One of the main uses of multicorrelated simulated random processes is in solving nonlinear random vibration problems by numerical integration of the governing differential equations. The response of a nonlinear string to a distributed noise input is presented as an example.
\end{abstract}

Subject Classification: $45.40 ; 40.30,40.22,40.35$.

\section{INTRODUCTION}

Many problems in mechanical and structural vibration can be included in the random vibration category. This category includes such problems as the response of aircraft to aerodynamic noise and turbulent boundary layers, the response of ground vehicles to rough roadways, and the response of tall buildings to wind loads. The forcing functions in these problems fluctuate in a random manner and in general only the statistical parameters which describe them are known. The theory of the linear response of structures subjected to random loads is well developed, ${ }^{1-3}$ however, in many cases the response of structures is not linear. For example, this can occur if the structural materials exhibits a nonlinear stressstrain relationship or if large deflections bring in-plane forces into play.

There are several techniques for handling nonlinear random vibration problems. The better known techniques are (1) the application of the Fokker-Planck equation (Markov vector approach), (2) the perturbation method, and (3) the method of equivalent linearization. ${ }^{1,3 \rightarrow}$ More recently, several investigators have used a method of computer simulation to solve nonlinear random vibration problems. ${ }^{7-10}$ Shinozuka's method consists of simulating a set of correlated random processes followed by numerical integration of the governing differential equations. This technique is very useful because it overcomes some of the limitations of the other techniques. For example, problems where the nonlinearities involve velocities as well as displacements, problems involving correlated exciting forces, and problems in which the input spectra are not white cannot be solved by the Fokker-Planck equation. ${ }^{4}$ Likewise, the perturbation and equivalent linearization techniques are limited to problems where the nonlinearities are small. These limitations do not apply to the direct simulation method.

The solution of anything more than a single-degreeof-freedom system by simulation requires that one be able to simulate correlated random processes. The work in this field has been done by Borgman, ${ }^{7}$ Shinozuka, ${ }^{8,9}$ and Hoshiya. ${ }^{10}$ Borgman used a filtered white-noise method. Shinozuka and Hoshiya both used trigonometric series, Shinozuka with random phase and Hoshiya with random amplitude. The technique suggested in this paper is based upon generating discrete frequency functions which correspond to the Fourier transform of the time series, and then using the fast Fourier transform (FFT) algorithm to obtain the actual time series. The advantages of this method over the other methods are its simplicity and its speed. We demonstrate that the computation speed can be more than an order of magnitude faster for this method.

In order to demonstrate one of the uses of multicorrelated random processes, we have included as an example the problem of a nonlinear string subjected to a distributed random input. The correlated processes, which we simulated in this case, were the generalized forces for the first three modes. The generalized responses of the first three modes were then found by numerical integration, and from these the rms displacements at the center of the string was obtained.

\section{SIMULATION TECHNIQUE}

Before we discuss the simulation technique, we wish to review some common notation used in digital data analysis. Let the time increment between discrete points in a time series be $h$, and let the total number of points in the series be $N$. The Nyquist folding frequency for such a sample is $1 / 2 h .{ }^{11}$ That is, the highest frequency that can be investigated if you sample a time series at intervals of length $h$ is $1 / 2 h$. The total length of the sample is $N h$, and the usual frequency increment for FFT data analysis is $1 / \mathrm{Nh}$. We will use $n h$ for our running value of time, and $k / N h$ as our running value of frequency.

The starting point in simulating a set of $M$ correlated time series is the cross-spectral density functions of the processes we wish to simulate. We need to know 
the power spectral density of each process and the cross-spectral density between all the processes. These spectral density functions are usually arranged in the form of a matrix appropriately called the cross-spectral density matrix or simply the spectral matrix. We let $G_{p g}(k / N h)$ represent the one-sided cross-spectral density function between process $x_{p}(n h)$ and process $x_{g}(n h)$, and we assume that the spectral matrix can be factored into a lower triangular matrix $\left[H_{p g}(k / N h)\right]$ and its complex transpose. That is, we assume $\left[H_{p g}(k / N h)\right]$ exists such that

$$
\left[G_{p g}(k / N h)\right]=\left[H_{p g}(k / N h)\right]\left[H_{p g}^{*}(k / N h)\right]^{T},
$$

where ${ }^{*}$ denotes the complex conjugate and $T$ the transpose. It is a fairly straightforward operation to find the elements $H_{p g}$ as outlined in Refs. 8 and 9. Equation 1 can be written in summation notation as

$$
\begin{aligned}
& G_{p g}(k / N h)=\sum_{i=1}^{p} H_{p i}(k / N h) H_{g i}(k / N h), \\
& p, g=1,2, \ldots, M .
\end{aligned}
$$

We now demonstrate that a set of discrete time series can be simulated using the following model:

$$
x_{p}(n h)=\frac{1}{N} \sum_{k=0}^{N-1} X_{p}(k / N h) \exp \left(j \frac{2 \pi k n}{N}\right),
$$

where $X_{p}(k / N h)$ are complex random numbers which are explained below. Note that $x_{p}(n h)$ is just the Fourier transform of $X_{p}(k / N h)$. The FFT algorithm is used to find $x_{p}(n h)$ for $n=0,1,2, \ldots, N-1$ in one step, instead of doing the summation indicated in Eq. 3 for each $n$. To obtain the terms $X_{p}(k / N h)$ we first generate a set of completely independent Gaussian random numbers $\zeta_{i k}$ $=\xi_{i k}+j \eta_{i k}$, using a standard random number generator subroutine, such that

$$
E\left[\xi_{i k}\right]=E\left[\eta_{i k}\right]=0
$$

and

$$
E\left[\xi_{l_{k}}^{2}\right]=E\left[\eta_{i k}^{2}\right]=0.5,
$$

where $E$ is the expectation operator. Then

$$
X_{p}(k / N h)=\left(\frac{N}{2 h}\right)^{1 / 2} \sum_{i=1}^{p} H_{p i}(k / N h) \zeta_{i k}
$$

or, in matrix form,

$$
\left\{\begin{array}{c}
X_{1} \\
X_{2} \\
\vdots \\
X_{M}
\end{array}\right\}=\left(\frac{N}{2 h}\right)^{1 / 2}\left(\begin{array}{cccc}
H_{11} & 0 & \cdots & 0 \\
H_{21} & H_{22} & \cdots & 0 \\
\vdots & \vdots & \cdots & \vdots \\
H_{M 1} & H_{M 2} & \cdots & H_{M M}
\end{array}\right)\left\{\begin{array}{l}
\zeta_{1 k} \\
\zeta_{2 k} \\
\zeta_{M k}
\end{array}\right\} .
$$

The terms $X_{p}(k / N h)$ have an additional property that is necessary to point out. If we take the inverse Fourier transform of Eq. 3, we get

$$
X_{p}(k / N h)=\sum_{n=0}^{N-1} x_{p}(n h) \exp \left(-j \frac{2 \pi k n}{N}\right) .
$$

It follows that

$$
X_{p}\left(\frac{N-k}{N h}\right)=\sum_{n=0}^{N-1} x_{p}(n h) \exp \left(j \frac{2 \pi k n}{N}\right) .
$$

Hence, if $x_{p}(n h)$ is real,

$$
X_{p}\left(\frac{N-k}{N h}\right)=X_{p}^{*}(k / N h) .
$$

That is, the terms $X_{p}$ for $N / 2<k \leq N-1$ are found from the terms for $0 \leq k \leq N / 2$.

Next we would like to demonstrate that the time series given by Eq. 3 do indeed have the proper power and cross-spectral densities. The cross correlation between time series $x_{p}(n h)$ and $x_{g}(m h)$, as given by Eq. 3, is

$$
\begin{aligned}
R_{p g}(m, n)=E\left\{\frac{1}{N^{2}} \sum_{k=0}^{N-1} \sum_{l=0}^{N-1}\right. & X_{p}(k / N h) X_{\xi}^{*}(l / N h) \\
& \left.\times \exp \left[j \frac{2 \pi(k n-l m)}{N}\right]\right\},
\end{aligned}
$$

where, since $x_{g}(m h)$ is a real function, it has been replaced by its complex conjugate. Because only the $X_{p}$ and $X_{B}$ terms are random in nature, the expectation operator can be brought inside the summation. If this is done, Eq. 9 reduces to

$$
\begin{aligned}
R_{p g}(m, n)= & \frac{1}{N^{2}} \sum_{k=0}^{N-1} \sum_{l=0}^{N-1} E\left[X_{p}(k / N h) X_{g}^{*}(l / N h)\right] \\
& \times \exp \left[j \frac{2 \pi(k n-l m)}{N}\right] .
\end{aligned}
$$

Considering Eq. $5 \mathrm{a}$ and the properties of $\zeta_{i k}$, it follows that

$$
\begin{aligned}
E\left[X_{p}(k / N h) X_{g}^{*}(l / N h)\right]= & \frac{N}{2 h} \sum_{i=1}^{p} \sum_{j=1}^{p} H_{p i}(k / N h) H_{s j}^{*}(l / N h) \\
& \times E\left[\zeta_{i k} \zeta_{3 l}^{*}\right] \\
& =\frac{N}{2 h} \sum_{i=1}^{p} H_{p i}(k / N h) H_{g i}(l / N h) \delta_{k l},
\end{aligned}
$$

where $\delta_{k l}$ is the Kronecker delta function. From Eqs. 10 and 11 we get

$$
\begin{aligned}
R_{p g}(m, n)= & \frac{1}{2 N h} \sum_{k=0}^{N-1} \sum_{i=1}^{p} H_{p i}(k / N h) H_{g i}(k / N h) \\
& \times \exp \left[j \frac{2 \pi k(n-m)}{N}\right] .
\end{aligned}
$$

The right-hand side ot Eq. 12 is dependent upon only the difference between $m$ and $n$, but not on their separate values. From this we conclude that the time series generated by this technique are stationary.

If we take the Fourier transform of Eq. 12 in order to obtain the cross-spectral density between processes $p$ and $g$, and multiply by a factor of two to get a onesided spectrum, we obtain Eq. 2. This confirms that the time series given by Eq. 3 does have the required power and cross-spectral densities.

The random processes generated by Eq. 3 are Gaussian. This follows from the central-limit theorem, the fact that the $\zeta_{i k}$ terms are Gaussian, and because Eq. 3 and Eq. 5 a are simple summations. 
As has been pointed out several times, the FFT algorithm is used to perform the Fourier transform of Eq. 3. The most common forms of this algorithm, such as the Cooley-Tukey procedure, require that the number of data points $N$ in a sample function be a power of two. To perform a simulation by the technique described here, it is necessary to chop the spectral density functions up into $N / 2$ slices, evaluate the $H_{p g}$ at each of these points, find the $X_{p}(k / N h)$ at each of these points, find the $X_{p}(k / N h)$ for $N / 2<k \leq N-1$ from $\mathrm{Eq} .8$, and finally carry out the Fourier transform of Eq. 3 using the FFT algorithm.

Most of the computation speed of this method of simulation comes from using the FFT algorithm. The speed ratio between evaluating $M(M+1) / 2$ series of $N$ cosine terms (as required by Shinozuka's method) and taking the FFT of $M$ series each with $N$ terms (as required by the method suggested here) is

$$
\text { speed ratio }=\frac{N}{4 P} \frac{M+1}{2},
$$

where $P=\log _{2} N{ }^{11}$ For example, if we wish to simulate ten series each with $2^{12}=4096$ time points, then the speed ratio is

$$
\text { speed ratio }=\frac{4096}{4(12)} \frac{(10+1)}{2} \simeq 470 .
$$

This speed ratio is based on the number of addition and multiplication operations that the computer must carry out.

\section{NONLINEAR STRING}

In order to demonstrate one of the uses of correlated random processes, we present here a Monte Carlo solution for the responses of a nonlinear string due to a distributed noise input. Although this example can be found elsewhere, ${ }^{8}$ we think it is worth repeating here in order to show how the correlated generalized forces can be simulated using our method. The governing differential equation for such a nonlinear string is

$$
\rho \frac{\partial^{2} u}{\partial t^{2}}+C \frac{\partial u}{\partial t}=\left[T_{0}+\frac{A E}{2 L} \int_{0}^{L}\left(\frac{\partial u}{\partial t}\right)^{2} d x\right] \frac{\partial^{2} u}{\partial x^{2}}+g(x, t)
$$

where $\rho$ is the mass per unit length, $u$ the displacement, $c$ the damping coefficient, $T_{0}$ the initial tension, $E$ the elastic modulus, $A$ the string's cross-sectional area, $L$ the string's length, and $g(x, t)$ the random distributed load. We have assumed that the damping is linear, viscous, and uniformly distributed along the string. Our boundary conditions are

$$
u(0, t)=u(L, t)=0 \text {. }
$$

We assume that the solution can be expanded in terms of the normal modes of a linear string which satisfy Eq. 16, that is,

$$
u(x, t)=\sum_{n=1}^{\infty} u_{n}(t) \sin (n \pi x / L) .
$$

If we substitute Eq. 17 into Eq. 15, multiply through by $\sin (m \pi x / L)$, and integrate over the length of the string, we get

$$
\begin{aligned}
\ddot{u}_{m} & +\frac{c}{\rho} \dot{u}_{m}+\left[\frac{T_{0}}{\rho}+\frac{A E}{4 \rho L} \sum_{k=1}^{\infty} u_{k}^{2}\left(\frac{k \pi}{L}\right)^{2}\right]\left(\frac{m \pi}{L}\right)^{2} u_{m} \\
& =\frac{2}{\rho L} \int_{0}^{L} g(x, t) \sin \left(m \pi_{x} / L\right) d x .
\end{aligned}
$$

This is a set of coupled, nonlinear differential equations. The correlated forces that we simulated to solve this problem are the generalized forces on the right-hand side of Eq. 18.

Our first step is to find the power and cross-spectral densities for the generalized forces. Let

$$
\begin{aligned}
& g_{m}(t)=\int_{0}^{L} g\left(x_{1}, t\right) \sin \left(m \pi x_{1} / L\right) d x_{1}, \\
& g_{n}(t+\tau)=\int_{0}^{L} g\left(x_{2}, t+\tau\right) \sin \left(n \pi x_{2} / L\right) d x_{2} .
\end{aligned}
$$

Then the cross correlation between these two generalized forces is

$$
\begin{aligned}
R_{m n}(\tau)= & \int_{0}^{L} \int_{0}^{L} R\left(\tau, x_{1}, x_{2}\right) \\
& \times \sin \left(m \pi x_{1} / L\right) \sin \left(n \pi x_{2} / L\right) d x_{1}, d x_{2},
\end{aligned}
$$

where $R\left(\tau, x_{1}, x_{2}\right)$ is the cross correlation between $g\left(x_{1}, t\right)$ and $g\left(x_{2}, t\right)$. Taking the Fourier transform of both sides of Eq. 20 gives

$$
\begin{aligned}
G_{m n}(f)= & \int_{0}^{L} \int_{0}^{L} G\left(f, x_{1}, x_{2}\right) \\
& \times \sin \left(m \pi x_{1} / L\right) \sin \left(n \pi x_{2} / L\right) d x_{1}, d x_{2},
\end{aligned}
$$

where $f$ is the frequency in hertz. Thus, if we know the

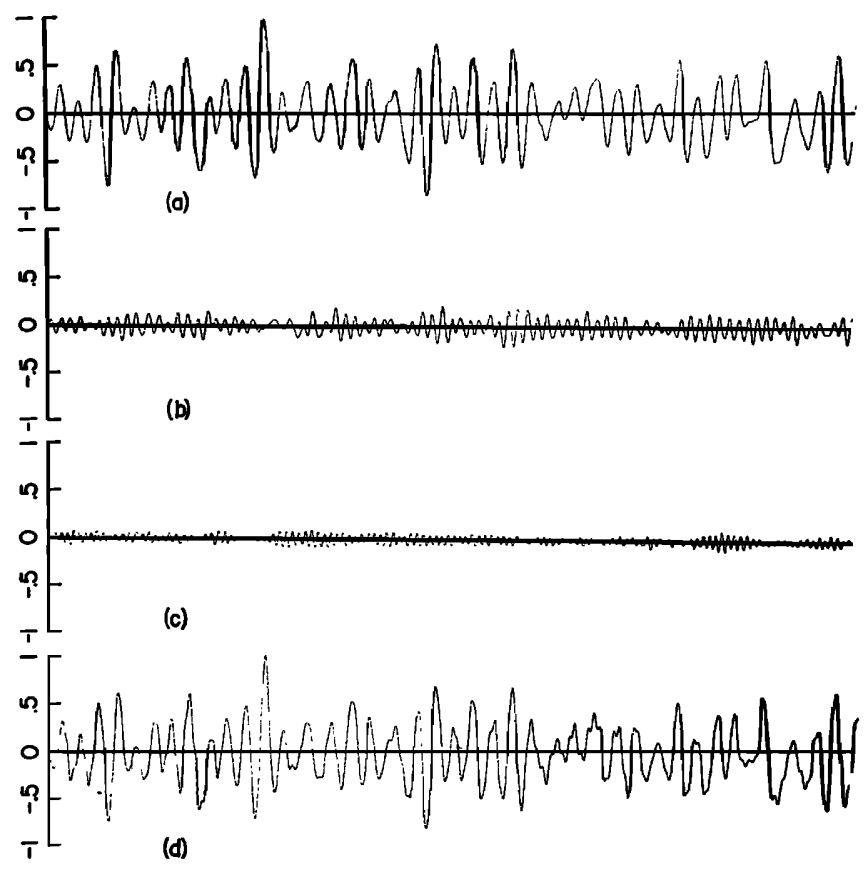

FIG. 1. Displacement time histories for a nonlinear string. (a) nondimensional generalized displacement for the first mode; (b) nondimensional generalized displacement for the second mode. (c) nondimensional generalized displacement for the third mode; and (d) nondimensional displacement time history at the center of the string. 


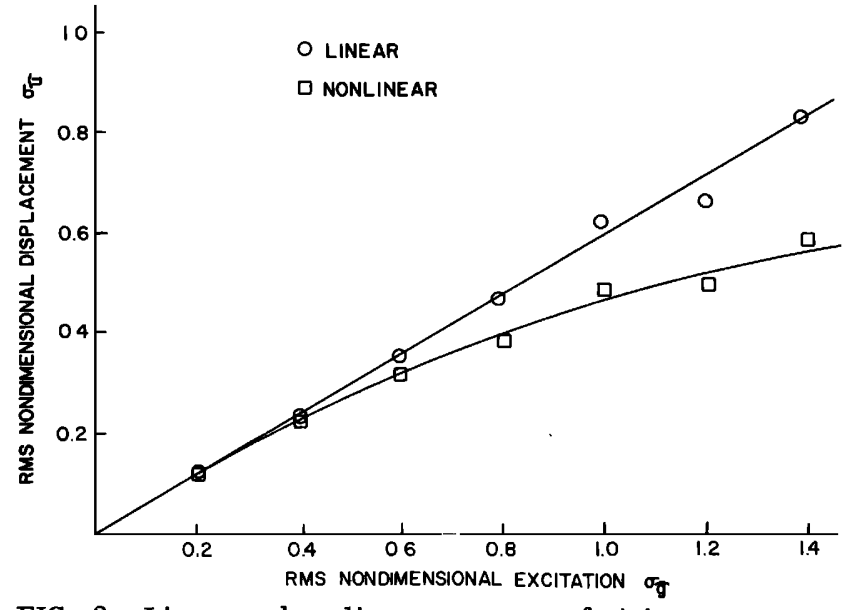

FIG. 2. Linear and nonlinear response of string.

cross-spectral density between the forces at any two points on the string, we can find the cross-spectral density between the generalized forces, and then go on to simulate these forces.

For this example, so that we could compare our work with Shinozuka's work, ${ }^{8}$ we used the following spectral density function:

$$
G\left(f, x_{1}, x_{2}\right)=\frac{2 \sigma^{2}}{\pi}\left(\frac{a}{a^{2}+4 \pi^{2} f^{2}}\right) \exp \left(-2 \pi f\left|x_{1}-x_{2}\right|\right),
$$

where $a$ and $\alpha$ are constants, and $\sigma^{2}$ is the mean-square value of the force at any point along the string. The next step is to substitute $G\left(f, x_{1}, x_{2}\right)$ from Eq. 22 into Eq. 21 and carry out the integration. This gives a somewhat cumbersome result, although the integration itself is fairly straightforward. The result is

$$
G_{m n}(f)=\frac{2 \sigma^{2}}{\pi}\left(\frac{a}{a^{2}+4 \pi^{2} f^{2}}\right)(A+B C),
$$

where

$$
\begin{aligned}
& A=\frac{2 \pi \alpha f L}{(2 \pi \alpha f)^{2}+(n \pi / L)^{2}} \delta_{m n}, \\
& B=\frac{m n \pi^{2} / L^{2}}{\left[(2 \pi \alpha f)^{2}+(m \pi / L)^{2}\right]\left[(2 \pi \alpha f)^{2}+(n \pi / L)^{21}\right.}, \\
& C=1+(-1)^{m+n}+\left[(-1)^{m+1}+(-1)^{n+1}\right] \exp (-2 \pi \alpha f L) .
\end{aligned}
$$

Having obtained the above expression for the crossspectral densities, we proceeded to simulate the generalized forces. We then solved the set of coupled, nonlinear equations numerically using a predictor-corrector difference-equation method. Our example was carried out for the case where $c / \rho=0.4 \pi / \mathrm{sec}, a=4 \pi /$ sec, $\alpha L=0.70 \mathrm{sec}, T_{0} / A E=0.050, L=25$ in. , $T_{0}=100$ lb, $\rho=0.040 \mathrm{lb} / \mathrm{in}$., and $E=30.10^{6} \mathrm{psi}$. Because the generalized forces drop off quickly with higher mode number and because we were looking for a rms displacement instead of a velocity or an acceleration, we considered only the first three modes.

The solution to Eq. 18 for a single set of generalized forces is shown in Fig. 1. The top three curves show the response of the first three modes, and the bottom curve shows the response at the midpoint of the string.
Figure 2 shows how the rms nondimensional response at the midpoint of the string is related to the rms nondimensional excitation force. These nondimensional quantities are defined by

$$
\begin{aligned}
& \hat{u}(L / 2, t)=u(L / 2, t) /(L / 10), \\
& \hat{g}(x, t)=g(x, t) /\left(T_{0} / L\right)
\end{aligned}
$$

The rms value of $\hat{g}(x, t)$ is independent of $x$. Also shown in Fig. 2 is the response of a linear string which we found using the above technique and dropping the nonlinear terms in Eq. 18. The linear string problem was done to satisfy ourselves that the computer programs were working properly. For a more detailed explanation of our work on this problem see Ref. 12.

\section{CONCLUSION}

We have developed a new method for simulating sets of multicorrelated random processes with specified cross-spectral densities. This method is both straightforward and fast. For large sets of correlated processes, it is orders of magnitude faster than other methods. This method gains its speed by using the FFT algorithm to obtain the random processes. We have used this method of simulation to study the random vibration of a nonlinear string. This technique of solving nonlinear random vibration problems overcomes most of the drawbacks inherent in other methods, as mentioned in the Introduction.

\section{ACKNOWLEDGMENT}

This work was supported by the National Aeronautics and Space Administration. The authors would like to express their appreciation to Dr. H. W. Tieleman of the Department of Engineering Science and Mechanics at Virginia Polytechnic Institute for his help and encouragement.

*Present address: Bolt Beranek and Newman, Inc., Cambridge, MA 02138.

†Present address: United Engineers and Constructors, 1401 Arch St., Philadelphia, PA.

${ }^{1}$ S. H. Crandell, Ed., Random Vibration (MIT Press, Cambridge, MA, 1963), Vol. 2.

${ }^{2}$ S. H. Crandall and W. D. Mark, Random Vibration in Mechanical Systems (Academic, New York, 1963).

${ }^{3}$ Y. K. Lin, Probabilistic Theory of Structural Dynamics (McGraw-Hill, New York, 1967).

${ }^{4}$ T. K. Caughey, "Derivation and Application of the FokkerPlanck Equation to Discrete Nonlinear Dynamic Systems Subjected to White Random Excitation," J. Acoust. Soc. Am. 35, 1683-1692 (1963).

${ }^{5} \mathrm{~S}$. H. Crandall, "Perturbation Techniques for Random Vibration of Nonlinear Systems," J. Acoust. Soc. Am. 35, 17001705 (1963).

${ }^{6}$ T. K. Caughey, "Equivalent Linearization Techniques," J. Acoust. Soc. Am. 35, 1706-1711 (1963).

${ }^{7}$ L. E. Borgman, "Ocean Wave Simulation for Engineering Design," J. Waterways Harbors Div., Proc. Am. Soc. Civil Eng. 95, No. WW4, 557-583 (1969).

${ }^{8} \mathrm{M}$. Shinozuka, "Simulation of Multivariate and Multidimensional Random Processes," J. Acoust. Soc. Am. 49, 357367 (1971).

${ }^{9} \mathrm{M}$. Shinozuka and C. J. Jan, "Simulation of Multivariate and Multidimensional Processes $\Pi$," Tech. Rep. No. 12, Dept. 
Civil Eng. and Eng. Mech., Columbia U., New York (1971).

${ }^{10} \mathrm{M}$. Hoshiya, L. E. Wittig, and A. K. Sinha, "Simulation of Multicorrelated Time Series," VPI-E-72-18, Dept. Eng. Sci. Mech., Virginia Poly. Inst. and State U. (1972).

${ }^{11}$ J. S. Bendat and A. G. Piersol, Random Data: Analysis and Measurement Procedures (Wiley-Interscience, New York, 1971).

${ }^{12} \mathrm{~A}$. K. Sinha, "On the Simulation of Multicorrelated Random Processes and its Application," Ph.D. thesis, Dept. Eng. Sci. Mech., Virginia Poly. Inst. and State U. (July 1973). 